\title{
Komplikationen nach distaler Radiusfraktur
}

\author{
Tim Lögters, Joachim Windolf
}

\section{Zusammenfassung}

Die Komplikationsrate nach einer distalen Radiusfraktur wird zwischen 6 und $80 \%$ angegeben. Komplikationen können eine Folge der Verletzung selbst oder der Behandlung sein. Die wesentlichen Komplikationen in der Versorgung distaler Radiusfrakturen sind Nervenirritationen, Beuge- und Strecksehnenläsionen, Infektionen, chronisch regionale Schmerzsyndrome (CRPS) sowie Ausheilungen in Fehlstellung. Die Gesamtkomplikationsrate für die mittlerweile weitreichend etablierte palmare Plattenosteosynthese ist deutlich geringer als für die Stabilisierung durch Bohrdrähte und/ oder den Fixateur externe. Die mit der palmaren Plattenosteosynthese assoziierten Komplikationen sind jedoch häufig technischer Art und können mit schwerwiegenden Konsequenzen für die Patienten verbunden sein.

\section{Complications of Distal Radius Fractures}

The complication rate after a distal radius fracture ranges between 6 and $80 \%$. Complications may result from the injury itself or the treatment. The major complications of distal radius fractures are the chronic regional pain syndrome (CRPS), nerve irritation, flexor and extensor tendon lesions and malunion. The overall complication rate for the now widely established volar plate fixation is much lower than for the stabilisation by K-wires and/or the external fixator. The complications associated with the volar plate fixation are frequently of a technical nature and associated with serious consequences for the patient.

\section{Einleitung}

Nahezu jeder 6. Bruch, der in einer Notaufnahme behandelt wird, ist eine distale Radiusfraktur. Trotz deren Häufigkeit steht die Behandlung nach wie vor unter besonderer Diskussion. Behandlungsziel sind die Wiederherstellung einer schmerzfreien uneingeschränkten Funktion des Handgelenks und die Vermeidung typischer Komplikationen. Es besteht ein weitgehender Konsens darüber, dass das Behandlungsziel am ehesten durch Wiederherstellung der Anatomie erreicht wird [5].

Komplikationen nach distalen Radiusfrakturen können eine Folge der Verlet-

OP-JOURNAL 2012; 28: 262-267

(c) Georg Thieme Verlag KG Stuttgart · New York DOI http://dx.doi.org/10.1055/s-0032-1327993

zung selbst oder der Behandlung sein und werden nach dem Schweregrad in „Minor“- und „Major“-Komplikationen mit und ohne Notwendigkeit einer chirurgischen Intervention unterteilt. Die Komplikationsrate nach distaler Radiusfraktur wird in der Literatur sehr variabel zwischen 6 und 80\% angegeben $[2,3,7]$. Die wesentlichen Komplikationen in der Versorgung distaler Radiusfrakturen sind Nervenirritationen, Beuge- und Strecksehnenläsionen, Infektionen, chronisch regionale Schmerzsyndrome (CRPS) sowie Ausheilungen in Fehlstellung. Im Fall des Auftretens ist für jede dieser Komplikationen in Abhängigkeit der Ausprägung eine deutliche Minderung des funktionellen Ergebnisses zu erwarten. Die nachhaltige und weitreichende Etablierung der palmaren winkelstabilen Plattenosteosynthese zur Versorgung der distalen Radiusfraktur hat zu einem Wandel der Komplikatio- nen in Art und Häufigkeit des Auftretens für die Frakturentität geführt [9,11]. Die Gesamtkomplikationsrate ist bei der offenen Reposition und Stabilisierung durch eine palmare Platte deutlich geringer als bei der Fixierung durch Bohrdrähte und/oder den Fixateur externe [4]. Während für die Bohrdrahtosteosynthese und den Fixateur externe Komplikationsraten von bis zu 50\% beschrieben sind, liegt diese bei der palmaren Plattenosteosynthese zwischen 7 und 30\% [4]. In diesem Übersichtsartikel sollen die wesentlichen Komplikationen in Abhängigkeit der Behandlungsart dargestellt werden.

\section{Nervenirritationen}

Eine Affektion peripherer Nerven nach distaler Radiusfraktur kann eine Folge der Fraktur selbst oder deren Behandlung sein. Die Inzidenz liegt bei bis zu $17 \%$ [7]. Während iatrogene Nervenverletzungen eher eine seltene Komplikation darstellen, treten neurologische Symptome auf der Basis eines Kompressionsschadens der Nerven häufig auf.

Aufgrund seiner Lage in unmittelbarer Nachbarschaft zum distalen Speichenende ist der $\mathrm{N}$. medianus der am häufigsten betroffene Nerv. Die Symptome können sich akut nach dem Trauma, verzögert nach einer operativen Stabilisierung oder spät nach Ausheilung der Fraktur manifestieren.

Ursächlich für ein akutes Karpaltunnelsyndrom (KTS) können ein ausgeprägtes Frakturhämatom oder dislozierte Frakturen sein. Milde Symptome eines KTS sind in bis zu einem Viertel aller Patienten mit einer distalen Radiusfraktur vorhanden. Unter Ruhigstellung in einer Gipsschiene und Hochlagerung treten die Symptome zumeist nur temporär auf und bedürfen keiner sofortigen weiterführenden Maßnahmen. In 5-9\% der Fälle ist jedoch aufgrund einer ausgeprägten klinischen Symptomatik eine 


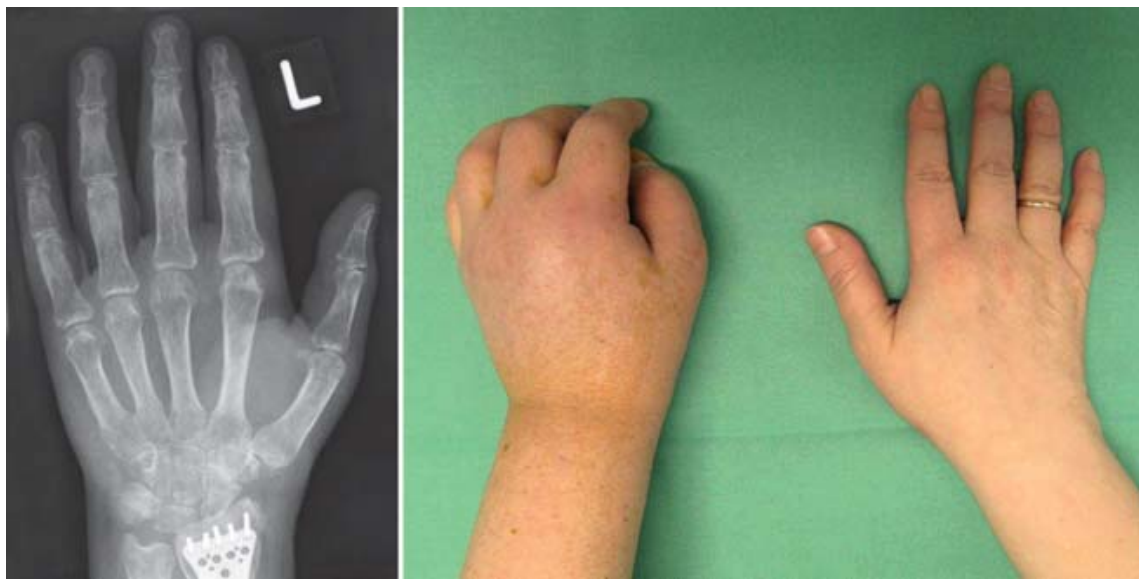

Abb. 1 Radiologisches und klinisches Bild eines chronisch regionalen Schmerzsyndroms (CRPS) 10 Wochen nach operativer Versorgung einer distalen Radiusfraktur mit einer palmaren winkelstabilen Platte.

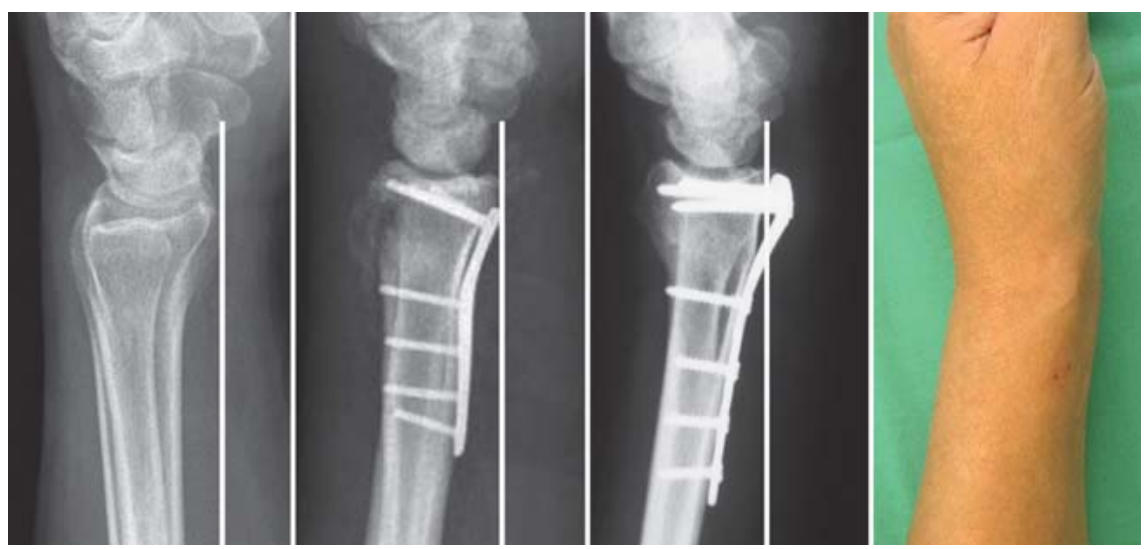

Abb. 2 Darstellung der „kritischen Linie“ tangential zum am weitesten palmar gelegenen Anteil des Radius und parallel zur palmaren Kortikalis des Radiusschafts in der seitlichen nativradiologischen Projektion des Handgelenks als Surrogat für die Watershed-Linie. Markierung bei einem unverletzten Handgelenk sowie nach Implantation einer palmaren Platte mit und ohne Berücksichtigung dieser Landmarke. Bild einer klinisch radiopalmar prominenten palmaren Platte durch eine zu distale Platzierung am distalen Radius.

sofortige Dekompression notwendig. Das Risiko für die Entwicklung dieser ausgeprägten frühen posttraumatischen KTS ist insbesondere bei Hochrasanztraumata und multifragmentären Frakturen deutlich erhöht.

Das Risiko, im Anschluss an eine operative Stabilisierung einer distalen Radiusfraktur ein Karpaltunnelsyndrom zu entwickeln, variiert in Abhängigkeit des Osteosyntheseverfahrens. Die Symptome können sich hierbei bereits früh nach der Operation, aber auch verzögert nach einem Intervall von Wochen manifestieren. Während für die Bohrdrahtosteosynthese mit oder ohne Fixateur externe das Risiko für die Entwicklung eines KTS als gering eingeschätzt wird, liegt die Inzidenz von Karpaltunnelsyndromen nach palmarer Plattenosteosynthese bei 0,5-22\% [7]. Als objektive Risikoindika- höht ist. Wir empfehlen in der Regel keine routinemäßige Dekompression des Karpaltunnels und stellen die Indikation nur in besonderen Fällen. Zu den besonderen Indikationen gehören präoperative Beschwerden, die mit dem klinischen Bild eines Karpaltunnelsyndroms einhergehen, oder ausgeprägte Fragmentdislokationen, die eine Einengung erwarten lassen. Ursächlich für eine verzögerte oder späte Manifestation eines KTS nach distaler Radiusfraktur können eine in Fehlstellung verheilte Fraktur, eine ausgeprägte Kallusformation oder eine prolongierte Ruhigstellung in Flexionsstellung des Handgelenks sein. Die kausale Therapie des späten postoperativen KTS besteht in der Dekompression des Karpalkanals und - in Abhängigkeit von der knöchernen Konsolidierung der frühzeitigen Entfernung der Platte oder ggf. der Korrektur einer etwaigen Fehlstellung.

Die wesentlichen Komplikationen in der Versorgung distaler Radiusfrakturen sind Nervenirritationen, Beuge- und Strecksehnenläsionen, Infektionen, chronisch regionale Schmerzsyndrome (CRPS) sowie Ausheilungen in Fehlstellung.

Die Gesamtkomplikationsrate ist für die palmare Plattenosteosynthese geringer als für die Bohrdrahtosteosynthese mit oder ohne Fixateur externe.

\section{Chronisch regionales Schmerzsyndrom (CRPS)}

Die Entwicklung eines CRPS nach distaler Radiusfraktur ist mit einem schlechten subjektiven und objektiven Behandlungsergebnis assoziiert (Abb. 1). Die Inzidenz des chronisch regionalen Schmerzsyndroms (CRPS) nach Verletzungen der oberen Extremität wurde in der Vergangenheit zwischen 8 und 35\% angegeben [7]. Zunehmende Erkenntnisse über die Pathophysiologie der Erkrankung und daraus abgeleiteter Maßnahmen zur Verhinderung haben zu einer deutlichen Reduktion von CRPS auch nach distalen Radiusfrakturen geführt. Neben einer individuellen Prädisposition wird eine Reihe beeinflussbarer Faktoren für die Entstehung eines CRPS angesehen. Prädisponierende Faktoren sind ein höheres Lebensalter und vorbestehende psychiatrische Erkrankungen. $\mathrm{Zu}$ den beeinflussbaren Faktoren zählen ausgeprägte posttraumatische oder postoperative Schmerzen, wiederholte Repositionsmanöver unter unzureichender Analgesie und eingeschränkte Bewegun- 
gen des Handgelenks über einen längeren Zeitraum. Das höchste Risiko für die Entwicklung eines CRPS besteht für die konservative Behandlung oder die Therapie mit einem Fixateur externe. Ein zu eng angelegter Gips oder eine zu starke Distraktion des Handgelenks über den Fixateur kann die Ursache für ein CRPS darstellen. Aus diesem Grund empfehlen wir die Dynamisierung eines Fixateur externe 2 Wochen nach primärer Anlage durch kurzzeitige Lockerung der Schraubenstangenfixierung. Seit weitreichender Anwendung der palmaren Platte als übungsstabile Osteosynthese mit frühfunktioneller Mobilisation des Handgelenks sind CRPS nach distaler Radiusfraktur seltener geworden. Die Inzidenz liegt mittlerweile bei unter $3 \%[2,7]$. Lediglich bei frühzeitigen Re-Osteosynthesen, Implantatfehllagen und nach Korrekturosteosynthesen besteht ein erhöhtes Risiko für ein CRPS auch bei der Anwendung einer palmaren Platte. In der Regel wird von operativen Maßnahmen in akuten Stadien des CRPS abgeraten. In Fällen jedoch, bei denen eine plattenassoziierte Komplikation wie z.B. eine $\mathrm{zu}$ weit distale Lage der palmaren Platte als Ursache für ein CRPS in Betracht kommt, kann durch einen frühzeitigen Eingriff in den frühen Phasen 1 oder 2 mit Rekonstruktion der anatomischen Verhältnisse der weitere Krankheitsverlauf des CRPS positiv beeinflusst werden. Ebenfalls eine Indikation zur frühzeitigen operativen Korrektur besteht beim CRPS als Folge einer in Fehlstellung verheilten distalen Radiusfraktur.

\section{Beugesehnenirritationen}

Die Irritation von Beugesehnen ist eine systemimmanente Komplikation der palmaren Platte am distalen Radius und tritt nach Bohrdraht- und dorsalen Plattenosteosynthesen sowie dem Fixateur externe nicht auf. Die Inzidenz von Beugesehnenrupturen nach palmarer Plattenosteosynthese am distalen Radius liegt in verschiedenen Studien zwischen 0 und 12\% [4]. Ursächliche Faktoren für Beugesehnenrupturen sind das Implantatdesign, prominente Schraubenköpfe und eine $\mathrm{zu}$ distale Positionierung der Platte.

Als Landmarke zur Positionierung einer Platte am palmaren Radius dient die von Orbay erstmals beschriebene „watershed line“ (englisch für Wasserscheide oder Wendepunkt) [6]. Die Watershed-Linie entspricht keiner anatomischen Struk-

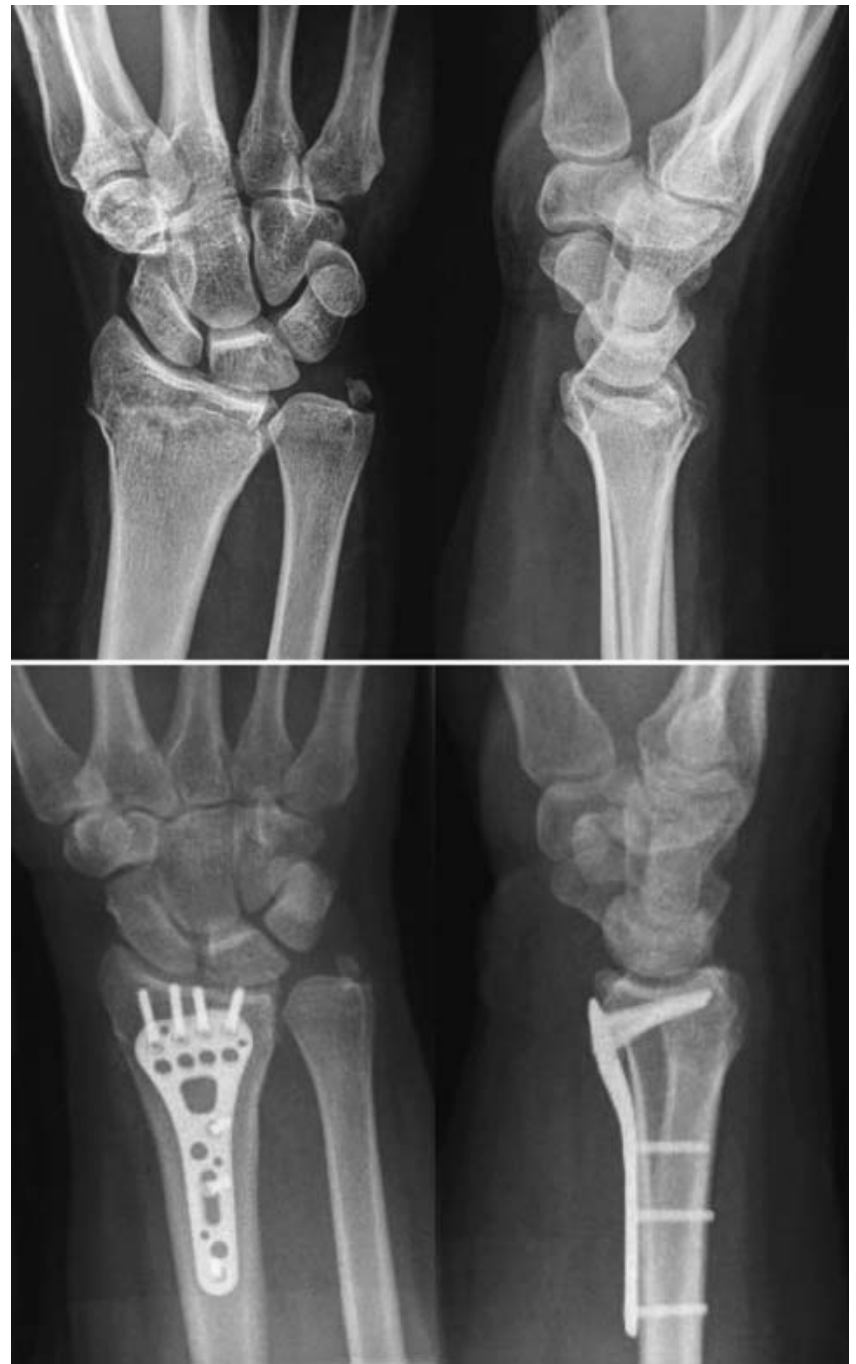

Abb. 3 Versorgung einer distalen Radiusfraktur durch eine palmare winkelstabile Platte mit flachem Profil. Es besteht eine gute Stabilisierung der ulnaren Säule durch eine distale Platzierung bei gleichzeitiger Berücksichtigung der Watershed-Linie im radialen Anteil des Radius in Projektion auf den Verlauf der FPL-Sehne.

tur, sondern beschreibt vielmehr den am weitesten palmar liegenden Aspekt der palmaren distalen Radiuskante. Bei einer Plattenpositionierung distal der Watershed-Linie nimmt der Kontaktdruck auf die Beugesehnen in Abhängigkeit von der Extensionsstellung des Handgelenks signifikant zu. Als radiologisches Surrogat für die Watershed-Linie kann in der seitlichen nativradiologischen Projektion des Handgelenks eine Linie tangential zum am weitesten palmar gelegenen Anteil des Radius parallel zur palmaren Kortikalis des Radiusschafts eingezeichnet werden [10]. Den höchsten Drücken ausgesetzt ist die Sehne des M. flexor pollicis longus (FPL), am ehesten bedingt durch ihre Lage und ihren Verlauf. Dies spiegelt sich auch in der klinischen Erfahrung wider, da die FPLSehne die am häufigsten von Rupturen betroffene Beugesehne nach palmarer Plattenosteosynthese ist. In der Mehrzahl der veröffentlichten Studien waren die Beugesehnenrupturen mit einer Plat- tenlage distal der Watershed-Linie assoziiert. Inwiefern neuere Plattendesigns mit einem nach distal flach auslaufenden Plattenprofil das Risiko von Beugesehnenrupturen reduzieren, bleibt abzuwarten (Abb. 3).

\section{Strecksehnenirritationen}

Bereits aufgrund des Verletzungsmechanismus kann es bei einer distalen Radiusfraktur zu einer traumatischen Schädigung der Strecksehnen kommen. Am häufigsten betroffen ist die lange Daumenstrecksehne (Extensor pollicis longus, EPL) [1]. Die Beeinträchtigungen der Strecksehnen können früh, aber auch verzögert nach dem Trauma auftreten und reichen von Sehnenirritationen bis hin zu Rupturen. Gerade bei gering dislozierten Radiusfrakturen wird aufgrund eines erhöhten Druckes unterhalb des unverletzten Retinaculum extensorum mit konsekutiver Minderperfusion der Strecksehnen das Risiko von zweizeiti- 

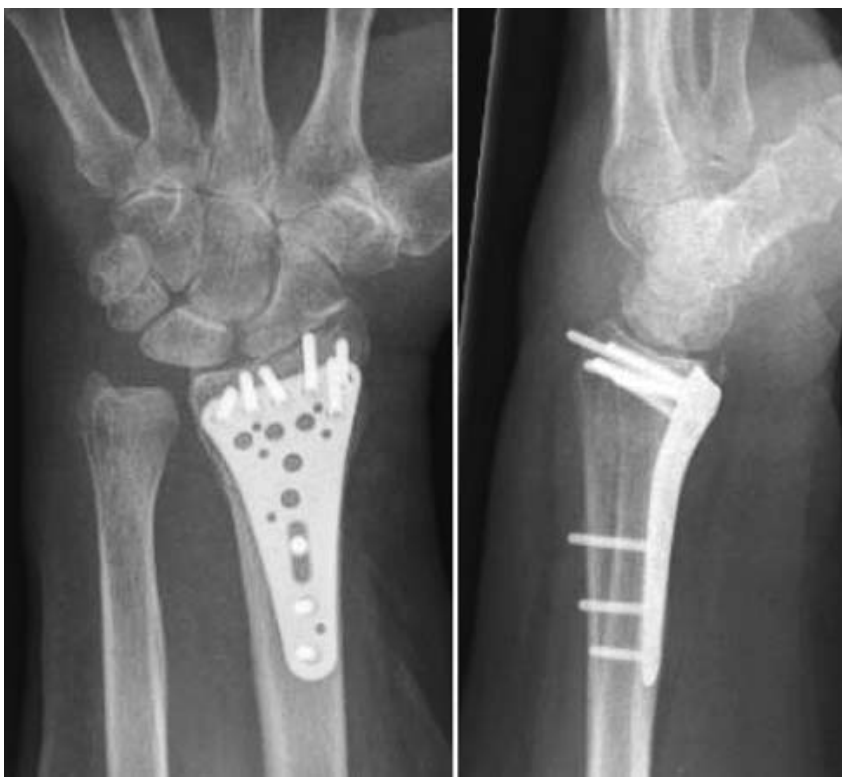

Abb. 4 Nativradiologische Bilder von an der dorsalen Kortikalis des distalen Radius überstehenden Schrauben einer palmaren Platte mit hohem Risiko einer Strecksehnenirritation und/oder -ruptur. gen Rupturen als vergleichsweise hoch eingeschätzt. Mit einer Inzidenz von ca. $1 \%$ handelt es sich bei der Ruptur zwar insgesamt um eine seltene Komplikation, jedoch mit potenziell schwerwiegenden Folgen für die betroffenen Patienten [2].

Auch Implantate können in Abhängigkeit von ihrer Lage zu einer Beeinträchtigung der Strecksehnen auf Höhe des Handgelenks führen. Die Strecksehnenruptur und -irritation ist die wesentliche Komplikation bei der Anwendung der dorsalen Plattenosteosynthese am distalen Radius. Eine Vielzahl an Techniken zur Vermeidung dieser Problematik wie z.B. die Bildung von retinakulären Interpositionslappen ist beschrieben, ohne jedoch das Risiko von Strecksehnenrupturen nach dorsaler Plattenosteosynthese nachhaltig und sicher zu reduzieren.

Bei ihrer Einführung wurde die Vermeidung von Strecksehnenirritationen als ein wesentlicher Vorteil der palmaren Plattenosteosynthese aufgeführt. Mit zunehmender Anwendung der palmaren Platte wurden als eine der behandlungsspezifischen Komplikationen Strecksehnenirritationen bis hin zu Rupturen durch an der dorsalen Kortikalis überstehende Schraubenspitzen oder intraoperative direkte Schädigung durch den Bohrvorgang beschrieben (Abb.4). Die Angaben zur Häufigkeit der Strecksehnenrupturen nach palmarer Plattenosteosynthese variieren zwischen 4,4 und $8,6 \%[2,3]$. Mit Abstand am häufigsten betroffen ist die Sehne des M. extensor pollicis longus (EPL). Für die EPL-Sehne ist aufgrund ihres anatomischen Verlaufs das Rupturrisiko besonders hoch. Sie verläuft im 3. Strecksehnenfach in einer engen „Sehnengrube“ unmittelbar ulnar des Tuberculum listeri. Diese enge und unflexible Führung sowie die vergleichsweise geringe Blutversorgung machen die EPL-Sehne besonders anfällig. Die direkte intraoperative Beurteilung eines etwaigen Überstands bzw. der Prominenz einer Schraubenspitze an der dorsalen Kortikalis ist nicht möglich. Wir empfehlen daher für die gelenknahe Plattenfixierung kürzere, sicher unikortikale Schrauben zu verwenden, da die Stabilität der Osteosynthese am distalen Radius mehr durch eine adäquate Schraubenlage, subchondral parallel zur Gelenkebene, als durch die Schraubenlänge selbst gewährleistet ist.

\section{Infektionen}

Die oberflächliche Infektion an der Eintrittsstelle von Bohrdrähten oder Schanz'schen Schrauben der Fixateure ist die häufigste Komplikation dieser beiden Osteosyntheseverfahren. Sie tritt in bis zu $15 \%$ bei den Bohrdrähten und bis zu 40\% der Fixateure auf [7]. Zumeist sind die Infektionen auf die Oberfläche begrenzt und können ohne Revision oder vorzeitige Implantatentfernung durch regelmäßige Pinpflege beherrscht werden. Die Notwendigkeit einer vorzeitigen Implantatentfernung aufgrund eines tief greifenden Infekts liegt in weniger als 5\% der Fälle vor. Das Risiko für Infektionen nach palmarer Plattenosteosynthese wird zwischen 0,3 und $2 \%$ angegeben [7]. Die Therapie dieser implantatassoziierten Infektionen ist abhängig vom Manifestationszeitpunkt und entspricht den Regeln der septischen Chi- rurgie mit potenziell nachhaltigen Folgen für das radiologische und funktionelle Ergebnis für die Patienten.

Komplikationen nach palmarer Plattenosteosynthese sind häufig technischer Art und können mit schwerwiegenderen Folgen für die Patienten einhergehen.

\section{Fehlverheilung}

Ursächlich für die Ausheilung einer distalen Radiusfraktur in Fehlstellung ist eine unzureichende Reposition und/oder Retention der Fraktur. Durch den Umbruch in der operativen Versorgung mit signifikant besseren radiologischen Resultaten mithilfe der palmaren Platte konnte die Rate der Fehlstellungen zwar deutlich reduziert werden, nach wie vor handelt es sich jedoch um eine der häufigsten Komplikationen mit einer Inzidenz von bis zu 17\% [8].

Die Ausheilung einer distalen Radiusfraktur in Fehlstellung hat nachhaltigen Einfluss auf die Gelenkgeometrie mit v.a. einer durch die Radiusverkürzung bedingten vermehrten Druckbelastung auf das ulnare Handgelenkkompartiment. Folgen können in Abhängigkeit von der Ausprägung Schmerzen, Einschränkung der Handgelenkbeweglichkeit, Verminderung der Griffkraft und vorzeitige Arthrose sein. Auch wenn Achsabweichungen nach distalen Radiusfrakturen nicht zwingend zu Beschwerden beim Patienten führen, besteht eine Korrelation zwischen klinischer Symptomatik und radiologischem Befund („function follows form“). Die Indikation zur operativen Korrektur stellen wir primär auf der Basis des klinischen Beschwerdebilds sowie der Funktion des Handgelenks und sekundär anhand der Veränderungen im Röntgenbild. Die therapeutischen Optionen umfassen Operationen mit dem Ziel der alleinigen Schmerzreduktion oder der Funktionsverbesserung mit und ohne Aufhebung der Fehlstellung. Die Zielsetzung einer Radiuskorrekturosteotomie liegt in der Wiederherstellung der knöchernen Verhältnisse mit dem Ziel, die Funktion zu verbessern und die Beschwerden zu reduzieren. Es ist daher von hoher Relevanz, im Vorfeld der Korrektur zu prüfen, inwiefern die erhobenen Beschwerden mit dem radiologischen Befund korrelieren und ob die alleinige knöcherne Wiederherstellung eine Reduktion der Beschwerden erwarten lässt. 
Die Radiuskorrektur wird idealerweise durch eine aufklappende („open-wedge“) Osteotomie erzielt. Lediglich bei sehr ausgeprägten Verkürzungen ist eine zuklappende Osteotomie des Radius in Kombination mit einer Ulnaverkürzungsosteotomie notwendig. Während in der Vergangenheit eine Vielzahl an Osteosyntheseverfahren zur Stabilisierung der Osteotomie angewandt wurde, haben sich in jüngerer Vergangenheit auch zur Korrekturosteosynthese winkelstabile Plattensysteme durchgesetzt. Die winkelstabilen Platten sind in der Lage, die hohen Kräfte, die von der Hand auf den Radius übertragen werden, zu neutralisieren. Die bei anderen Osteosyntheseverfahren zur Defektauffüllung zumeist notwendigen Spongiosatransplantationen sind daher in der Regel bei Anwendung der palmaren Platte nicht notwendig. Bei erheblicher Verkürzung des Radius mit sehr großen Defekten nach Längenwiederherstellung ohne Kontakt zwischen den Hauptfragmenten besteht aber weiterhin die Indikation zur Interposition eines kortikospongiösen Spanes (Abb. 5).

\section{Implantatentfernung}

Während die Entfernung von Bohrdrähten und des Fixateur externe immer zeitgerecht nach knöcherner Konsolidierung durchgeführt und dadurch eine weiterführende Mobilisation des Handgelenks erst ermöglicht wird, bestehen für die Entfernung von palmaren Platten am distalen Radius keine besonderen Indikationen. Gemäß Leitlinien sollte die Indikation individuell und abhängig von Alter und Aktivitätsgrad des Patienten, Material und Rigidität des Implantats sowie lokalen Beschwerden gestellt werden. Aktuell wird diskutiert, die Empfehlung zur Entfernung einer palmaren Platte am distalen Radius großzügig und vergleichsweise früh nach einer Operation auszusprechen. Diese Einschätzung begründet sich im Wesentlichen in der Sorge um eine Affektion der Beugesehnen. Da jedoch die Inzidenz der Beugesehnenrupturen nach palmarer Plattenosteosynthese mit $2-12 \%$ objektiv gering ist, kann allein daraus die generelle Empfehlung zur Implantatentfernung nicht gefordert werden. Die Indikation zur Entfernung sollte ausschließlich auf der Basis von klinischen und/oder radiologischen Kriterien gestellt werden, die ein höheres Risiko für eine Beugesehnenruptur nahelegen.

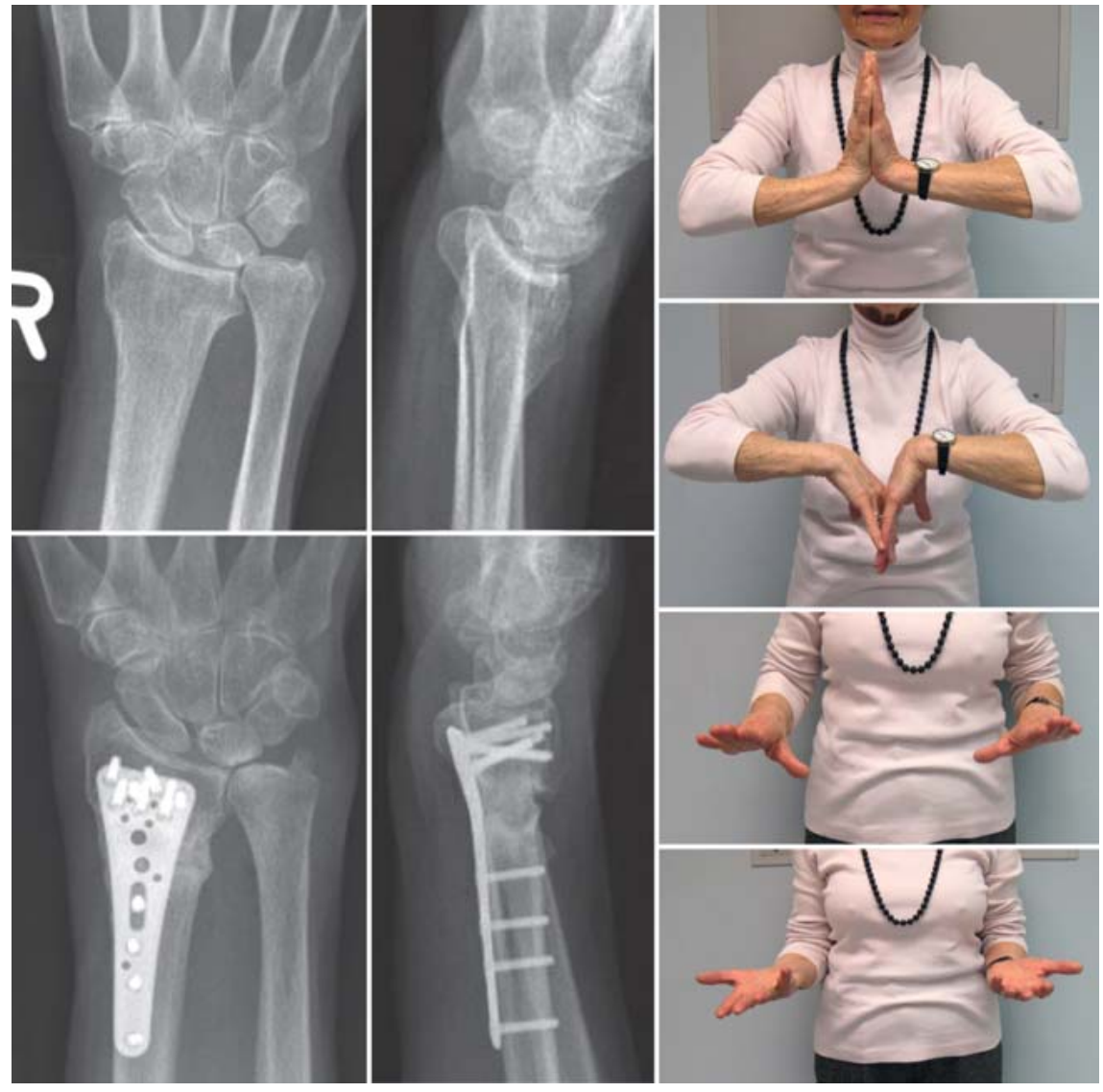

Abb. 5 Fehlverheilte distale Radiusfraktur mit wesentlicher Verkürzung und dorsopalmarer Reklination des distalen Radius. Radiologisches und klinisches Resultat nach Korrekturosteotomie, Defektauffüllung mit einem kortikospongiösen Span und Stabilisierung mit einer winkelstabilen palmaren Platte.

Als radiologisches Kriterium kann die Lagebeziehung der Platte zur WatershedLinie dienen. Hierbei wird die Indikation zur Entfernung der palmaren Platte bei Überragen der Watershed-Linie durch das distale Plattenende gestellt. Beschwerden der Patienten und klinische Befunde sind schwieriger zu objektivieren. In einigen Fällen ist die Platte am radialen Ende prominent bzw. tastbar, welches von den Patienten als störend empfunden wird.

Relevant für die Indikationsstellung zur Implantatentfernung ist zudem das Intervall zwischen Operation und Auftreten einer Beugesehnenruptur. Die Angaben hierzu unterliegen einer hohen Variabilität und werden zwischen 3 und 14 Monaten nach der Implantation angegeben. Die Mehrzahl aller Patienten berichten über Beschwerden im Sinne einer Tenosynovialitis unterschiedlicher Ausprägung im Vorfeld des eigentlichen Rupturereignisses. Auf der anderen Seite sind Fälle beschrieben, in denen eine Sehnenruptur auftrat, ohne dass klinisch nachweisbare Prodrome vorhanden wa- ren. Zweifelsohne sollte vor Entfernung der Platte die knöcherne Konsolidierung der Fraktur radiologisch gesichert sein.

An unserer Klinik empfehlen wir die Entfernung von palmaren Platten am distalen Radius frühestens 6 Monate nach der Implantation unter Berücksichtigung der folgenden Faktoren:

- Plattenlage in Bezug auf die Watershed-Linie

- Beschwerden aufgrund von Prominenz/Tastbarkeit der Platte

- Beschwerden im Sinne einer Beugesehnentenosynovialitis

- Lebensalter und Wunsch des Patienten

Die Indikation zur Entfernung sollte auf der Basis von klinischen und/oder radiologischen Kriterien gestellt werden, die ein höheres Risiko für eine Beugesehnenruptur nahelegen. Bei der Versorgung von distalen Radiusfrakturen mit der palmaren Platte sollte auf die korrekte Indikation und eine sachgerechte Anwendung geachtet werden. 


\section{Schlussfolgerungen}

Bei korrekter Durchführung besteht für die offene Reposition und interne Stabilisierung von distalen Radiusfrakturen mit der palmaren winkelstabilen Platte ein deutlich geringeres Gesamtkomplikationsrisiko als für die Bohrdrahtosteosynthese mit oder ohne Fixateur externe und die konservative Therapie. Es ist jedoch zu bedenken, dass die mit der palmaren Platte assoziierten Komplikationen zum einen häufig auf technische Fehler zurückzuführen sind und zum anderen von schwerwiegender Art und mit nachhaltiger Einschränkung für die Funktion des Handgelenks oder der Hand für die Patienten verbunden sein können. Trotz weitreichender Verfügbarkeit sollte daher auf eine sachgerechte Indikation und exakte Anwendung der palmaren Platte zur Versorgung von distalen Radiusfrakturen geachtet werden.

\section{Literatur}

${ }^{1}$ Al-Rashid M, Theivendran $K$, Craigen MA. Delayed ruptures of the extensor tendon secondary to the use of volar locking compression plates for distal radial fractures. J Bone Joint Surg [Br] 2006; 88: 1610-1612

2 Arora R, Lutz M, Hennerbichler A et al. Complications following internal fixation of unstable distal radius fracture with a palmar lockingplate. J Orthop Trauma 2007; 21: 316-322

3 Berglund LM, Messer TM. Complications of volar plate fixation for managing distal radius fractures. J Am Acad Orthop Surg 2009; 17: 369-377

${ }^{4}$ Diaz-Garcia RJ, Oda T, Shauver MJ et al. A systematic review of outcomes and complications of treating unstable distal radius fractures in the elderly. J Hand Surg Am 2011; 36: 824-35.e2

${ }^{5}$ Gehrmann SV, Windolf J, Kaufmann RA. Distal radius fracture management in elderly patients: a literature review. J Hand Surg Am 2008; 33: 421-429

${ }^{6}$ Orbay JL. The treatment of unstable distal radius fractures with volar fixation. Hand Surg 2000; 5: 103-112

7 Patel VP, Paksima N. Complications of distal radius fracture fixation. Bull NYU Hosp Jt Dis 2010; 68: 112-118
8 Prommersberger KJ, van Schoonhoven J. Corrective surgery after fractures of the distal radius. Unfallchirurg 2007; 110: 617-627

${ }^{9}$ Schädel-Höpfner M, Diener M, Windolf J. Gesicherte Literaturergebnisse zur Therapie distaler Radiusfrakturen. Trauma Berufskrankh 2008; 10: 230-235

10 Soong M, Earp BE, Bishop G et al. Volar locking plate implant prominence and flexor tendon rupture. J Bone Joint Surg [Am] 2011; 93: 328-335

11 Windolf J, Hakimi M, Schädel-Höpfner M. Ergebnisse der palmaren winkelstabilen Plattenosteosynthese am distalen Radius. Trauma Berufskrankh 2008; 10 (Suppl. 2): 236-240

\section{Priv.-Doz. Dr. Tim Lögters}

Oberarzt

Prof. Dr. med. Joachim Windolf Direktor

Klinik für Unfall- und Handchirurgie Universitätsklinikum Düsseldorf

Moorenstraße 5

40225 Düsseldorf

tim.loegters@med.uni-duesseldorf.de 